\title{
Coping style and depression influence the healing of diabetic foot ulcers: observational and mechanistic evidence
}

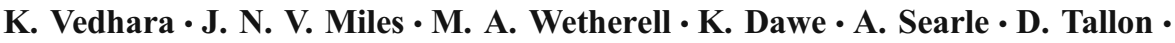 \\ N. Cullum • A. Day • C. Dayan • N. Drake • P. Price • J. Tarlton • J. Weinman • \\ R. Campbell
}

Received: 11 November 2009 /Accepted: 10 March 2010/Published online: 22 April 2010

(C) Springer-Verlag 2010

\begin{abstract}
Aims/hypothesis Experimental evidence suggests that the healing of diabetic foot ulcers is affected by psychosocial factors such as distress. We examined this proposal in a prospective study, in which we considered the role of psychological distress and coping style in the healing of diabetic foot ulcers over a 24 week period. We also explored the role of salivary cortisol and matrix metalloproteinases (MMPs) as potential mechanisms.

Methods For this prospective observational study we recruited 93 (68 men; mean age 60 years) patients with neuropathic or neuroischaemic diabetic foot ulcers from specialist podiatry clinics in secondary care. Clinical and
\end{abstract}

\section{K. Vedhara $(\bowtie)$}

Institute of Work, Health and Organisations, International House,

University of Nottingham,

Jubilee Campus,

Nottingham NG8 1BB, UK

e-mail: Kavita.Vedhara@nottingham.ac.uk

J. N. V. Miles

Rand Corporation,

Santa Monica, CA, USA

\section{A. Wetherell}

Department of Psychology, University of Northumbria,

Newcastle upon Tyne, UK

K. Dawe $\cdot$ R. Campbell

Department of Social Medicine, University of Bristol,

Bristol, UK

\section{A. Searle $\cdot$ D. Tallon}

Department of Community-Based Medicine, University of Bristol, Bristol, UK

\section{N. Cullum}

Department of Health Sciences, University of York,

York, UK demographic determinants of healing, psychological distress, coping, salivary cortisol and both MMP2 and MMP9 were assessed at baseline. Ulcers were assessed at baseline and at 6,12 and 24 weeks post-baseline. The primary outcome was ulcer status at 24 weeks, i.e. healed vs not healed.

Results After controlling for clinical and demographic determinants of healing, ulcer healing at 24 weeks was predicted by confrontation coping, but not by depression or anxiety. Patients with unhealed ulcers exhibited greater confrontation coping (model including depression: OR $0.809,95 \%$ CI $0.704-0.929, p=0.003$; model including anxiety: OR $0.810,95 \%$ CI $0.704-0.930, p=0.003$ ).

A. Day

Department of Cellular and Molecular Medicine,

University of Bristol,

Bristol, UK

C. Dayan

HW Labs for Integrative Neuroscience and Endocrinology,

University of Bristol,

Bristol, UK

N. Drake

Department of Podiatry, Southmead Hospital,

Bristol, UK

P. Price

School of Medicine, Cardiff University,

Cardiff, UK

J. Tarlton

Matrix Biology Research Group, University of Bristol,

Bristol, UK

J. Weinman

Health Psychology Section, Institute of Psychiatry,

London, UK 
However, change in ulcer size over the observation period was associated with depression only $(p=0.04, d=0.31)$. Healed ulcers by 24 weeks were also associated with lower evening cortisol, higher precursor MMP2 and a greater cortisol awakening response.

Conclusions/interpretation Confrontation coping and depression predict ulcer healing. Our preliminary enquiry into biological mechanisms suggests that cortisol and precursor MMP2 may underlie these relationships.

Keywords Coping $\cdot$ Cortisol $\cdot$ Depression .

Diabetic foot ulcers · Healing · MMPs · Prospective ·

Psychological distress

\section{Abbreviations \\ HPA Hypothalamic-pituitary-adrenal axis \\ MMP Matrix metalloproteinase}

\section{Introduction}

The prevalence of foot ulceration among patients with diabetes mellitus is between $4 \%$ and $10 \%$ [1]. The emotional, physical and financial costs are considerable, with foot ulcer patients reporting greater depression and poorer quality of life, while increased morbidity and mortality rates are estimated to cost health services $£ 220$ million per year [2-4]. The 5 year amputation and mortality rates for this patient group are $19 \%$ and $44 \%$ respectively [3]. These costs are exacerbated by slow healing rates, with as many as $67 \%$ of diabetic foot ulcers remaining unhealed after 20 weeks of care [5].

These poor clinical outcomes have driven enquiry into factors that influence healing, including psychosocial factors such as psychological distress [6]. This proposal emerged from pioneering research examining the relationship between psychological distress and the healing of experimental wounds in non-clinical populations. For example, acute examination stress has been associated with a $40 \%$ delay in the healing of punch biopsy wounds $[7,8]$. Similarly, chronic care-giving stress was associated with a $25 \%$ delay in the healing of similar wounds $[8,9]$. Such findings have prompted investigations into these relationships in connection with chronic wounds, such as diabetic foot ulcers. In this regard, Monami and colleagues [9] reported that ulcer healing (over 6 months) and ulcer recurrence (over 12 months) were associated with greater depression in diabetic patients. In contrast, Winkley et al. [10] failed to observe significant associations between depression and ulcer outcomes, but did report that baseline depression predicted mortality over 18 months in patients with foot ulcers. Similarly, a retrospective study of patients with a range of chronic wounds, including diabetic foot ulcers, reported that while depression was associated with ulcer healing over a 6 month period, the effect did not remain significant after adjustment for other variables [11].

Thus, the evidence regarding psychosocial factors and diabetic foot ulcer healing is modest and equivocal. Indeed, a recent meta-analysis identified a need for further research in this area and the consideration of a wider range of factors than simply indices of distress [12]. Accordingly, our primary aim was to examine the effects of psychological distress and coping styles on the healing of diabetic foot ulcers, after controlling for potential clinical and demographic determinants of healing. The assessment of coping styles, in addition to distress, was conducted because of their central role in the human stress process, and specifically their influence on the individual's emotional reactions to demanding/stressful experiences, including disease [13]. Indeed, in diabetes, the interaction between coping style and psychological distress has been shown to influence glycaemic control [14-16]. Our secondary aims included: (1) an examination of the effects of distress and coping style on an additional healing outcome, i.e. change in ulcer size over the observation period; and (2) an exploration of potential biological mechanisms, e.g. whether endocrine and biochemical variables that are affected by distress and might be expected to influence healing actually differed between patients whose ulcers healed and those whose ulcers did not heal. This was achieved by measuring salivary cortisol and the matrix metalloproteinases (MMPs) 2 and 9. Salivary cortisol captures the activity of the hypothalamic-pituitary-adrenal axis (HPA), the primary system mediating the relationship between psychological distress and immunity. The MMPs 2 and 9 are markers and mediators of connective tissue remodelling (MMP-2) and inflammatory cell infiltration (MMP-9), and are known to be linked to healing of dermal ulcers $[17,18]$. Both the precursor (pro-MMP) and activated (act-MMP) forms of these enzymes were measured.

It was hypothesised that coping styles and distress would be associated with a greater likelihood of ulcers not healing by 24 weeks (primary aim) and less change in ulcer size over the observation period (secondary aim). However, in view of the absence of previous related evidence, we did not specify a priori which coping styles would emerge as significant predictors. We further hypothesised that patients with unhealed ulcers by 24 weeks would exhibit significantly different levels of salivary cortisol, and of MMP-2 and MMP-9 at the site of the ulcer, when compared with patients with healed ulcers. Previous research suggests that distress is associated with dysregulation in the production of cortisol, leading to reduced and increased production of the hormone $[19,20]$. We did not, therefore, predict the direction of this relationship. In contrast, increased levels of MMPs has been associated with ulcer chronicity [18]. We 
therefore predicted that MMP levels would be greater in patients whose ulcers remained unhealed at 24 weeks.

\section{Methods}

Patients A convenience sample of patients with diabetes mellitus and a foot ulcer was recruited from outpatient podiatry clinics in secondary care between 2002 and 2008 . Patients were recruited into a longitudinal research programme examining psychological and behavioural aspects of diabetic foot ulceration. The clinical team identified eligible patients and introduced them to the research. Patients willing to find out more about the study and/or participate were then introduced to a member of the research team who, in turn, provided further written and verbal information. Written informed consent was obtained from all participating patients.

A total of 169 patients were recruited into the programme. Of these, 24 withdrew during follow-up and a further 33 were lost to follow-up. In the present article we report on data from 93 patients who contributed complete data for the primary analysis, i.e. patients for whom data on predictor variables were available at baseline and data on ulcer status at 24 weeks). Reasons for exclusion from the analysis were: (1) no clinically verified information on whether or not the ulcer had healed by the census date $(n=$ 25 ); and (2) missing data across one or more variables included in the primary analysis $(n=51)$. Details of the original cohort and the subgroup who provided complete data for the primary analysis are given in Table 1. One-way ANOVAs and $\chi^{2}$ analyses revealed (data not shown) that no differences were evident between the groups on the variables reported in this manuscript. Of the patients with complete data for the primary analysis, 56 had a healed ulcer by week 24 and 37 remained unhealed. Of those whose ulcer was unhealed, 14 had an amputation and three died during the follow-up period.

All hospital trusts approached regarding this study agreed to participate and ethical approval was obtained from all trusts. All clinics subscribed to a standard regimen of foot care, i.e. aggressive debridement at each visit, treatment of infections with antibiotics and the use of removable Scotch-casts and other footwear/devices for offloading ulcers on weight-bearing areas, minimising the likelihood of between-centre variations in treatment outcomes. Inclusion/exclusion criteria ensured the population consisted of patients with neuropathic or neuroischaemic ulcers. Patients were not eligible if they had: no palpable pulses on the affected foot; a history of major amputation (i.e. any lower limb amputation greater than a single digit); known large vessel peripheral vascular disease (e.g. previous bypass surgery, angioplasty); advanced diabetic retinopathy with
Table 1 Demographic and clinical characteristics of patients with and without complete data for primary analysis

\begin{tabular}{|c|c|c|}
\hline Characteristics & $\begin{array}{l}\text { Complete } \\
\text { data }\end{array}$ & $\begin{array}{l}\text { Incomplete } \\
\text { data }\end{array}$ \\
\hline Patients, $n$ & 93 & 76 \\
\hline \multicolumn{3}{|l|}{ Socio-economic factors } \\
\hline Age (years) & $60.7(10.97)$ & $61.56(12.64)$ \\
\hline Male sex $(n)$ & 68 & 60 \\
\hline White ethnicity ( $n$ ) & 86 & 54 \\
\hline \multicolumn{3}{|l|}{ Marital status $(n)$} \\
\hline Single & 14 & 11 \\
\hline Married/cohabiting & 60 & 24 \\
\hline Widowed & 6 & 4 \\
\hline Divorced/separated & 10 & 9 \\
\hline Other (not specified) & 3 & 28 \\
\hline \multicolumn{3}{|c|}{ Gross household income (annual) $(n)$} \\
\hline$<£ 5,000$ & 3 & 3 \\
\hline$£ 5,000-9,999$ & 20 & 12 \\
\hline$£ 10,000-14,999$ & 10 & 6 \\
\hline$£ 15,000-19,999$ & 17 & 6 \\
\hline$£ 20,000-24,999$ & 6 & 3 \\
\hline$£ 25,000-29,999$ & 3 & 2 \\
\hline$£ 30,000-34,999$ & 3 & 3 \\
\hline$£ 35,000-39,999$ & 2 & - \\
\hline$>£ 40,000$ & 7 & 2 \\
\hline Other (not specified) & 22 & 39 \\
\hline \multicolumn{3}{|l|}{ Clinical factors } \\
\hline Type 2 diabetes $(n)$ & 64 & 53 \\
\hline $\mathrm{HbA}_{1 \mathrm{c}}(\%)$ & $8.73(1.86)$ & $8.57(1.82)$ \\
\hline \multicolumn{3}{|l|}{ Ulcer status } \\
\hline Infected $(n)$ & 35 & 30 \\
\hline Healed by 24 weeks $(n)$ & 56 & 42 \\
\hline Baseline ulcer area $\left(\mathrm{mm}^{2}\right)$ & $17.22(27.69)$ & $15.91(27.98)$ \\
\hline Neuropathy score $(\%)$ & $73(31.1)$ & $75(31.2)$ \\
\hline Ischaemia score $(\%)$ & $69.5(35.2)$ & $72(35.1)$ \\
\hline Previous ulcers $(n)$ & $1.13(1.58)$ & $1.37(3.15)$ \\
\hline BMI $\left(\mathrm{kg} / \mathrm{m}^{2}\right)$ & $28.91(5.57)$ & $28.88(5.55)$ \\
\hline Arthritis present $(n)$ & 22 & 16 \\
\hline Asthma present $(n)$ & 6 & 2 \\
\hline Angina present $(n)$ & 12 & 5 \\
\hline Hypertension present $(n)$ & 45 & 22 \\
\hline Cancer present $(n)$ & 3 & 1 \\
\hline Heart disease present $(n)$ & 14 & 7 \\
\hline \multicolumn{3}{|l|}{ Psychological factors } \\
\hline Baseline anxiety & $6.88(5.02)$ & $5.18(4.08)$ \\
\hline Clinically anxious (\%) & 20 & 8.5 \\
\hline Baseline depression & $6.05(4.43)$ & $4.98(3.64)$ \\
\hline Clinically depressed (\%) & 16.7 & 6.8 \\
\hline Baseline confrontation coping & $18.26(3.96)$ & $18.02(3.67)$ \\
\hline Baseline avoidant coping & $13.14(2.91)$ & $13.59(3.28)$ \\
\hline Baseline acceptance coping & $6.44(2.42)$ & $6.45(2.41)$ \\
\hline
\end{tabular}

Values are mean (SD) unless indicated otherwise 
severe visual impairment; advanced nephropathy (e.g. on dialysis); other severe disabling medical conditions (e.g. stroke); or were being treated with platelet-derived growth factor, tissue engineered skin or total contact casts.

Patients participated in a prospective observational study conducted over 24 weeks and were assessed at baseline (week0) and weeks6, 12 and 24. Baseline measurements included: ulcer assessment, i.e. present/absent and ulcer area measured in square millimetres, glucose control, psychological distress and coping style. Data on the following were also recorded: neuropathy, ischaemia, presence/absence of ulcer infection (as reported in medical notes), BMI, number of previous ulcers, diabetes type, patients' self-report of other chronic conditions, age, sex, income, smoking and marital status. Ulcer assessments were repeated at weeks 6,12 and 24 .

Ulcer assessment Ulcer assessments involved determining: (1) whether or not the ulcer had healed; and (2) the size of unhealed ulcers at each follow-up. The former was determined with reference to clinical records. The latter involved using a transparent film and tracing the topical area of the wound. The tracing was then placed on a digital tablet (Visitrack: Smith and Nephew, London, UK) and the area of the ulcer was re-traced with a stylus to produce a measurement of absolute ulcer area. The timing of the ulcer assessments was dictated by patients' referral to the specialist podiatry clinics and entry into the study.

Glucose control Glycated haemoglobin $\left(\mathrm{HbA}_{1 \mathrm{c}}\right)$ was measured to provide a surrogate marker of disease control 2 to 3 months prior to study entry. $\mathrm{HbA}_{1 \mathrm{c}}$ was measured by cation exchange high-performance liquid chromatography using an analyser and associated reagents (HA-8140; A. Menarini Diagnostics, Wokingham, UK). The assay was maintained in line with the Diabetes Control and Complications method [21], with no significant assay drift and a between-batch imprecision (CV) of $1.8 \%$ (at mean $\mathrm{HbA}_{1 \mathrm{c}}$ $5.5 \%)$. All assays were performed on the same instrument.

Neuropathy, ischaemia and ulcer classification Neuropathy was assessed by applying a $10 \mathrm{~g}$ nylon monofilament to a number of sites on the affected foot and patients reporting the presence/absence of sensation. Level of neuropathy was based upon the number of tested sites with sensory loss. Consistent with established criteria [22], individuals were classified according to per cent of sensory loss as follows: $30 \%$ to $50 \%$ of tested sites 'mild neuropathy', $60 \%$ to $80 \%$ 'moderate neuropathy' and $90 \%$ to $100 \%$ 'severe neuropathy'. Percentage rather than absolute values were used, as the number of sites assessed varied between podiatrists. Ischaemia was assessed by measuring palpable pulses at the dorsalis pedis and posterior tibial areas of the affected foot. Ulcers were classified as neuropathic, i.e. sensory loss in $60 \%$ or greater of sites tested, or neuroischaemic (neuropathy combined with evidence of ischaemia).

Psychological distress and coping The Hospital Anxiety and Depression Scale assessed anxiety and depression [23]. Scores were converted into binary outcomes to reflect the presence (i.e. scores of 11 or greater) or absence (i.e. scores of 10 or less) of clinical anxiety and depression [24]. Cronbach's alpha reliability coefficients in the present study were 0.86 and 0.54 for anxiety and depression respectively.

The Medical Coping Modes Questionnaire [25] assessed confrontation, avoidance and acceptance-resignation coping. Confrontational styles are characterised as being more controlling, competitive and extroverted; avoidant styles demonstrate less knowledge and attention to their condition; acceptance-resignation styles exhibit greater helplessness and less affective expression. Cronbach's alpha reliability coefficients in the present study were 0.71 (confrontation coping), 0.54 (avoidance coping) and 0.73 (acceptanceresignation coping).

Salivary cortisol and MMPs Samples of saliva and wound fluid were collected at baseline for the measurement of cortisol and both MMP2 and MMP9. For cortisol, participants collected four saliva samples into tubes (Salivette; Sarstedt, Nümbrecht, Germany) over a single day, i.e. on waking, 30 min later, between 11:00 and 13:00 hours (before lunch), and between 20.00 and 22:00 hours (at least $2 \mathrm{~h}$ after evening meal). Participants were asked to not brush their teeth before providing the first two samples and not to consume food or caffeinated drinks in the hour before providing all samples. They were also asked to refrigerate samples until returned to the study site. Salivary cortisol was determined by ELISA (assays carried out by Cultech, Swansea, Wales, UK). The sensitivity of the assay was $0.3 \mathrm{nmol} / \mathrm{l}$ with an inter-assay variation of $3.43 \mathrm{nmol} / 1$ and an intra-assay variation of $2.66 \mathrm{nmol} / \mathrm{l}$.

Wound fluid was collected at baseline. Sterile, preweighed filter paper was applied to the wound to absorb fluid using methods described previously [18], and then reweighed and stored at $-80^{\circ} \mathrm{C}$ until processing. Levels of MMPs were normalised to the mass of fluid collected. The presence of MMP2 and -9 was detected in wound fluid samples using gelatine substrate SDS-polyacrylamide gel electrophoresis (zymography) as described previously [26-28].

Salivary samples for the measurement of cortisol and wound fluid samples for the measurement of MMPs were not collected from all patients. Their collection was dictated by whether appropriate funding and ethics approval had been obtained for these aspects of the study. Accordingly, 89 patients provided saliva samples for the measurement of cortisol (data available from $82,81,82$ and 85 patients for 
samples 1, 2, 3 and 4 respectively). One-way ANOVA and $\chi^{2}$ tests examined whether the cortisol subgroup was representative of the original cohort for the variables reported in this manuscript. This subsample only differed in the measure of acceptance coping, baseline $\mathrm{HbA}_{1 \mathrm{c}}$ and marital status, with patients who provided saliva samples having marginally lower $\mathrm{HbA}_{1 \mathrm{c}}$ levels (8.34 vs 9.10, $p=$ 0.011 ), a slightly greater propensity towards acceptanceresignation coping (6.83 vs 5.77, $p=0.014$ ) and being more likely to be single ( $22 \%$ vs $9 \%$ of patients). Cortisol awakening response was only calculated in patients who provided information on the specific times at which they had provided cortisol samples and who reported adherence to the protocol, i.e. first sample was taken on waking and the second one $30 \mathrm{~min}$ later $(n=45)$. One-way ANOVA compared the cortisol awakening response with that of patients providing all four cortisol samples. No statistically significant differences were observed.

Finally, wound fluid was collected from 21 patients at baseline. One-way ANOVAs and $\chi^{2}$ tests compared patients who provided samples with those who did not. The groups were largely comparable on all variables reported in this manuscript, except that patients who provided wound fluid samples were less likely to have an infected ulcer ( $20 \%$ vs $43 \%$ of patients; $p=0.020)$ and more likely to report high blood pressure $(69 \%$ vs $42 \%$ of patients; $p=0.013$ ).

Statistical methods For the primary analysis it was estimated that a sample size of 90 participants would provide $80 \%$ power to detect an odds ratio of 1.9 associated with a 1 standard deviation change in a normally distributed predictor variable, where the response probability at the mean of the predictor is 0.5 , using alpha $=0.05$. Testing the primary aim involved logistic regressions in which the binary outcome captured whether or not the ulcer had healed by the end of the observation period, i.e. by 24 weeks. For these analyses, patients were designated as 'not healed' if: (1) they were deemed to have an active ulcer at 24 weeks, i.e. one where clinical notes indicated that complete healing of the topical surface of the ulcer had not been achieved by the 24 week census point; (2) they had had an ulcer-related amputation during follow-up; or (3) they had died during follow-up. In contrast, patients for whom the podiatrists reported complete healing of the topical surface of the ulcer at some stage during follow-up were designated as 'healed'. The classification of deceased patients and those undergoing amputation as 'unhealed' is a convention reported by other groups [29].

Separate preliminary analyses were first conducted for each potential clinical and demographic determinant of ulcer healing, as measured at baseline, i.e. age, sex, marital status, income, ulcer infection, number of previous ulcers, type 1/type 2 diabetes, $\mathrm{HbA}_{1 \mathrm{c}}$, $\mathrm{BMI}$, neuropathy, ischaemia, smoking, chronic co-morbid conditions and ulcer area. A conservative cut-off of $p \leq 0.1$ was adopted for these variables to qualify for inclusion in the final model. Thus, the final model for the primary analysis included only those clinical and demographic factors that met the $p \leq 0.1$ criterion, and the measures of anxiety, depression and coping. Due to the intercorrelation between the measures of anxiety and depression $(r=0.674, p<0.0001)$, the model was run separately for these variables.

The secondary aim of exploring the effect of psychosocial factors on change in ulcer size over the observation period, involved repeated measures ANOVA. The dependent variables were the ulcer area measures at 0, 6, 12 and 24 weeks, and thus only included patients for whom ulcer area data were available at each time point. For patients who died or who had an amputation during the follow-up period, the last available ulcer measurement was used for all observations after death or amputation occurred. This approach is statistically conservative as it assumes no further change would have occurred in these patients.

Finally, one-way ANOVA analyses compared levels of cortisol and both MMP2 and -9 between patients who had healed vs not healed over the observation period. For cortisol, this involved comparisons across all four time points at which samples were collected and also looking at
Table 2 Results from final logistic regression model (primary analysis) examining role of clinical depression and coping style in the healing (healed vs not healed) of diabetic foot ulcers

Patients analysed, $n=93$

\begin{tabular}{lccc}
\hline Variables & Odds ratio & $p$ value & $95 \%$ CI \\
\hline Type 1/type 2 diabetes & 1.216 & 0.744 & $0.377-3.925$ \\
Ischaemia & 1.007 & 0.320 & $0.993-1.021$ \\
Angina & 3.488 & 0.215 & $0.484-24.115$ \\
Heart disease & 0.829 & 0.826 & $0.156-4.395$ \\
Ulcer area at baseline & 0.273 & 0.011 & $0.100-0.746$ \\
Clinical depression & 2.389 & 0.368 & $0.358-15.392$ \\
Confrontation coping & 0.809 & 0.003 & $0.704-0.929$ \\
Avoidant coping & 1.011 & 0.901 & $0.851-1.201$ \\
Acceptance-resignation coping & 1.071 & 0.633 & $0.809-1.417$ \\
\hline
\end{tabular}


Table 3 Results from final logistic regression model (primary analysis) examining role of clinical anxiety and coping in the healing (healed vs not healed) of diabetic foot ulcers

Patients analysed, $n=93$

\begin{tabular}{lccc}
\hline Variables & Odds ratio & $p$ value & $95 \%$ CI \\
\hline Type 1/type 2 diabetes & 1.039 & 0.947 & $0.333-3.241$ \\
Ischaemia & 1.008 & 0.295 & $0.993-1.022$ \\
Angina & 3.509 & 0.208 & $0.497-24.757$ \\
Heart disease & 0.923 & 0.925 & $0.176-4.834$ \\
Ulcer area at baseline & 0.292 & 0.015 & $0.109-0.784$ \\
Clinical anxiety & 2.293 & 0.280 & $0.509-10.324$ \\
Confrontation coping & 0.810 & 0.003 & $0.704-0.930$ \\
Avoidant coping & 1.104 & 0.876 & $0.852-1.206$ \\
Acceptance-resignation coping & 1.073 & 0.585 & $0.833-1.383$ \\
\hline
\end{tabular}

the difference between the first and second samples. This latter variable, referred to as the cortisol awakening response, is a widely used measure of HPA reactivity [30, 31].

All analyses were conducted using SPSS version 15 and non-normally distributed variables were subjected to logarithmic transformation.

\section{Results}

For all analyses, the final models were examined including and excluding deceased and amputated patients included in the 'not healed' group. The results were largely unaffected by the exclusion of these patients and so the data from the larger sample. i.e. deceased and amputated patients included, are presented. Where significant differences occurred, these are reported.

Preliminary logistic regressions revealed that type 1/type 2 diabetes, ischaemia, angina, heart disease and ulcer area at baseline all met the a priori cut-off of $p \leq 0.1$ for inclusion in the final model. Thus, the final model for the primary analysis included these variables, plus anxiety, depression and coping styles. Ulcer area at baseline and confrontation coping were observed to be significant predictors of healing, with patients reporting a greater propensity towards confrontation coping and exhibiting larger ulcers at study entry being less likely to have a healed ulcer by week 24 (Tables 2 and 3). The sensitivity analysis, i.e. exclusion of patients who had died or had an amputation during follow-up, produced comparable findings. The only significant change was that ulcer size at baseline was reduced to non-significant (OR 0.354, $p=0.116,95 \% \mathrm{CI}$ $0.097-1.291$ and OR 0.305, $p=0.094,95 \%$ CI 0.076-1.222 for anxiety and depression models respectively). The effects of confrontation coping were unchanged.

Secondary analyses examined the relationship of psychosocial factors with change in ulcer area over the observation period (with measures of anxiety and depression examined in separate models). As the primary analysis revealed that only confrontation coping was a significant predictor of healing, these analyses focussed on confrontation coping only. Neither anxiety nor confrontation coping predicted change in ulcer area and none of the interaction effects emerged as significant. However, depression was a statistically significant predictor, with patients exhibiting clinical depression having smaller changes in ulcer size over time (Table 4, Fig. 1). Indeed, clinical depression accounted for over $30 \%$ of the variance in ulcer size over the observation period.

The final analyses examined whether there were significant differences in MMP and cortisol levels between patients whose ulcers healed and those whose ulcers did not heal. One-way ANOVAs revealed that patients with unhealed ulcers by 24 weeks had lower levels of evening cortisol, higher levels of pro-MMP2 and a greater cortisol awakening response at baseline (Table 5).

\section{Discussion}

The present study examined the role of psychological distress and coping in the healing of diabetic foot ulcers. The primary analyses revealed that, after accounting for probable clinical and demographic determinants of healing, confrontation coping was associated with non-healing. However, secondary analyses revealed that confrontation coping was

Table 4 Results from repeated measures ANOVA examining between-participant effects of confrontational coping and clinical depression $(n=23)$ or clinical anxiety $(n=23)$ on change in ulcer area over the observation period

\begin{tabular}{llll}
\hline Variables & $F$ & $p$ value & $d^{\mathrm{a}}$ \\
\hline Depression & 5.30 & 0.04 & 0.31 \\
Confrontation coping & 0.462 & 0.860 & 0.235 \\
Depression $\times$ confrontation coping & 0.009 & 0.927 & 0.001 \\
Anxiety & 1.297 & 0.281 & 0.115 \\
Confrontation coping & 0.540 & 0.803 & 0.302 \\
Anxiety $\times$ confrontation coping & 0.451 & 0.722 & 0.119 \\
\hline
\end{tabular}

${ }^{\mathrm{a}} d=$ effect size 


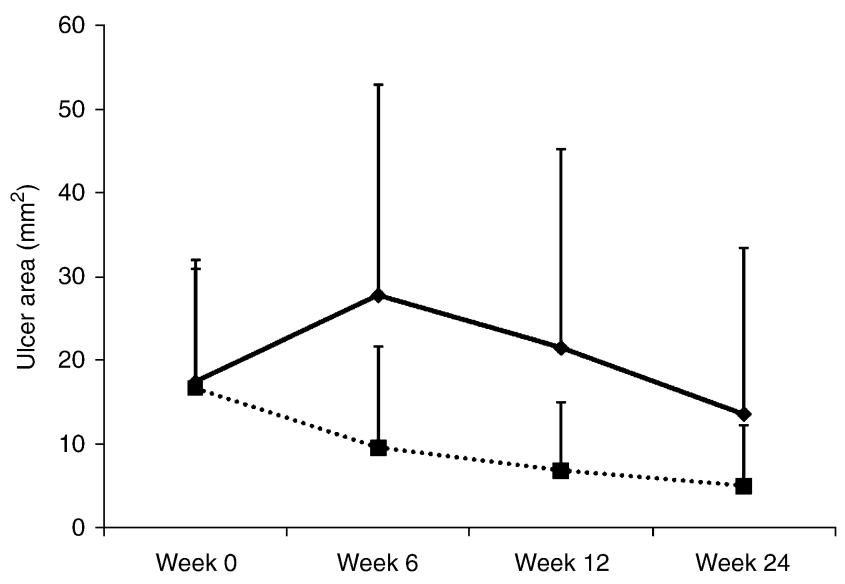

Fig. 1 Mean (and standard deviation) ulcer area scores over observation period for patients with and without clinical depression, i.e. with scores of $>11$ and $<11$ respectively on the depression subscale of the Hospital Anxiety and Depression Scale. Continuous line, score $>11$; dotted line, score $<11$

not related to the change in ulcer area over time. Instead, this was predicted by depression, with patients reporting clinical depression exhibiting a smaller change in ulcer area over time. Finally, analysis of potential mechanisms suggested that cortisol and pro-MMP2 levels may contribute to the relationship between psychosocial factors and diabetic foot ulcer healing.

The clinical implications of these data are potentially farreaching, suggesting that psychological interventions that reduce depression and promote effective coping could significantly improve healing rates in this patient group, thus reducing the likelihood of complications such as amputation and death. To our knowledge, only one published study has examined the effects of such an intervention in diabetic patients with foot ulceration. Its authors reported that $87.5 \%$ of patients receiving biofeedback-assisted relaxation training had a healed ulcer by the end of their observation period, compared with only $43.8 \%$ of patients who received 'usual care' [32].

Table 5 Results from one-way ANOVAs comparing cortisol and MMP indices in patients who healed or did not heal

${ }^{a}$ Values are mean $(\mathrm{SD})$
There are, however, several issues worthy of comment. The first concerns confrontation coping and how it might affect ulcer healing. According to the Medical Coping Modes Questionnaire authors [25], individuals with confrontational styles embody a controlling, extraverted and competitive nature. These patients are therefore more likely to challenge the advice of healthcare professionals and/or be less willing to adhere to treatment recommendations. This could suggest a behavioural mechanism, whereby confrontation coping results in behaviours that directly impact on healing. A physiological mechanism, however, is also possible in that previous research has indicated that characteristics associated with confrontation coping have been linked to physiological changes that might impact on wound healing $[33,34]$.

The second issue concerns the seemingly independent effects of confrontation coping and depression on ulcer healing (coping predicted ulcer resolution, depression predicted change in absolute size of the ulcer over time). This may simply reflect the fact that the categorical outcome offered a blunter assessment of changes in the ulcer over time, leading to the identification of different predictors. However, post-hoc analyses revealed that the measures of depression and confrontation coping were unrelated $(r=-0.02, p=\mathrm{NS}$; as were anxiety and confrontation coping: $r=-0.025, p=$ NS). This supports the possibility that these different psychosocial factors may have affected ulcer healing in different ways, a suggestion supported by Winkley et al., who observed that depression predicted mortality, but not ulcer recurrence or amputation [10].

The third issue relates to our preliminary analyses examining whether healed and unhealed patients had differences in cortisol and MMP2 and -9 levels. The cortisol findings suggest that patterns of HPA activity were different for evening cortisol and early morning peak measures. The direction of these relationships is somewhat in keeping with previous research. For example, greater early morning peak levels have been associated with greater depressive symp-

\begin{tabular}{|c|c|c|c|c|c|c|}
\hline \multirow{12}{*}{$\begin{array}{l}\text { Table } 5 \text { Results from one-way } \\
\text { ANOVAs comparing cortisol } \\
\text { and MMP indices in patients } \\
\text { who healed or did not heal }\end{array}$} & Variables & $n$ & Not healed ${ }^{\mathrm{a}}$ & Healed $^{\mathrm{a}}$ & $F$ & $p$ value \\
\hline & Cortisol (nmol/1) & & & & & \\
\hline & Time 1: on waking & 82 & $13.57(6.65)$ & $17.85(20.21)$ & 1.653 & 0.202 \\
\hline & Time 2: 30 min later & 81 & $16.09(8.09)$ & $22.00(34.69)$ & 0.391 & 0.534 \\
\hline & Time 3: $11: 00-13: 00$ hours $(n=82)$ & & $9.11(6.27)$ & $10.94(11.38)$ & 0.828 & 0.365 \\
\hline & Time 4: $20: 00-22: 00$ hours $(n=85)$ & & $6.10(4.33)$ & $9.76(15.42)$ & 3.850 & 0.053 \\
\hline & MMPs (\% of activity relative to standard) & & & & & \\
\hline & Precursor MMP2 & 21 & $7.15(5.32)$ & $3.99(3.79)$ & 4.990 & 0.039 \\
\hline & Activated MMP2 & 21 & $1.12(1.08)$ & $0.65(0.79)$ & 1.589 & 0.234 \\
\hline & Precursor MMP9 & 21 & $55.55(32.43)$ & $50.18(40.78)$ & 0.562 & 0.463 \\
\hline & Activated MMP9 & 21 & $8.89(8.18)$ & $8.80(19.23)$ & 0.424 & 0.533 \\
\hline & Cortisol early morning peak & 45 & $2.61(1.25)$ & $-0.38(-1.05)$ & 5.678 & 0.022 \\
\hline
\end{tabular}


toms [20]; and while lower evening cortisol levels are usually associated with fewer depressive symptoms [35], there is evidence that evening levels are indeed lower in individuals reporting burnout [36].

Baseline levels of pro-MMP2 were also increased in patients whose ulcers remained unhealed. Physiological activation of MMP2 is tightly regulated and precisely localised to the cell surface. Thus, levels of pro-MMP2 reflect the amount of enzyme available for activation. Higher levels in unhealed patients indicate over-activity of MMP2, resulting in greater breakdown of the extracellular matrix, thus hindering healing [17]. Indeed, increased expression and/or activity of MMPs, including MMP2, has previously been observed in diabetic foot ulcers and venous ulcers, and related to healing status [18]. Our findings are therefore consistent with the notion that increased levels of MMP2 may contribute to the chronicity of diabetic foot ulcers. Furthermore, as alterations in MMP2 have been related to biological indices of distress, i.e. noradrenaline (norepinephrine), cortisol [37], the differences in MMP2 levels may reflect a mechanism by which psychological factors influence healing.

Finally, some limitations should be acknowledged. First, our assessment of wound size was restricted to measuring the topical surface of the wound. Thus, our data cannot inform us as to the effects of psychosocial factors on healing processes below the skin. Second, a number of methodological factors may have influenced the generalisability and precision of our findings, in particular, the use of a convenience sample and one dominated by white participants. In addition, variable selection for the primary analysis was based on statistical significance. Although this resulted in the inclusion of explanatory variables previously shown to influence healing, e.g. baseline ulcer size, others such as age and sex were excluded [38]. Moreover, our secondary analyses were conducted on modest sample sizes. The third limitation is that the reliability data for the measures of depression and avoidance coping were modest. Finally, recruitment of patients occurred when they presented at specialist podiatry clinics in secondary care. There may, therefore, have been considerable variability between patients in ulcer duration and management prior to study entry.

Notwithstanding the above, the present study suggests that depression and coping style are associated with a greater likelihood of diabetic foot ulcers not healing over a 6 month period, albeit through seemingly independent pathways, and that cortisol and pro-MMP2 may be among the mechanisms underlying these relationships. Further research is required to elucidate these mechanisms and to develop interventions, in particular those geared to modifying coping style and distress, e.g. coping effectiveness training [39], in order to improve clinical outcomes in this patient group.
Acknowledgements The present study was funded by a programme grant from the Medical Research Council. The authors also wish to acknowledge the clinical teams and patients at all participating centres for their unwavering support and cooperation. The following individuals contributed to this research: B. Maitland: research administration (University of Bristol); A. Strode: patient recruitment and study design (Department of Health); N. Percy: patient recruitment (North Bristol NHS Trust); L. Gale: patient recruitment (University of Bristol); W. Grainger: patient recruitment (Gloucestershire Hospitals NHS Foundation Trust); E. Dougan: patient recruitment and study design (University Hospitals Bristol, NHS Foundation Trust); E.A.M. Gale: study design (North Bristol NHS Trust); E. Mudge: patient recruitment (Cardiff University); L. Higgs, C. Hurley and K. Harman: patient recruitment (Royal United Hospital Bath NHS Trust).

Duality of interest The authors declare that there is no duality of interest associated with this manuscript.

\section{References}

1. Singh N, Armstrong DG, Lipsky BA (2005) Preventing foot ulcers in patients with diabetes. JAMA 293:217-228

2. Vileikyte L (2001) Diabetic foot ulcers: a quality of life issue. Diabetes Metab Res Rev 17:246-249

3. Moulik PK, Mtonga R, Gill GV (2003) Amputation and mortality in new-onset diabetic foot ulcers stratified by etiology. Diabetes Care 26:491-494

4. O'Meara S, Cullum N, Majid M, Sheldon T (2000) Systematic reviews of wound care management: (3) antimicrobial agents for chronic wounds; (4) diabetic foot ulceration. Health Technology Assessment vol. 4, no. 21. Available from www.hta.ac.uk/fullmono/ mon421.pdf, accessed 15 May 2010

5. Kantor J, Margolis DJ (2000) Expected healing rates for chronic wounds. Wounds 12:155-158

6. Vileikyte L (2007) Stress and wound healing. Clin Dermatol 25:49-55

7. Marucha PT, Kiecolt-Glaser JK, Favagehi M (1998) Mucosal wound healing is impaired by examination stress. Psychosom Med 60:362-365

8. Kiecolt-Glaser JK, Marucha PT, Malarkey WB, Mercado AM, Glaser R (1995) Slowing of wound healing by psychological stress. Lancet 346:1194-1196

9. Monami M, Longo R, Desideri CM, Masotti G, Marchionni N, Mannucci E (2008) The diabetic person beyond a foot ulcerhealing, recurrence, and depressive symptoms. J Am Podiatr Med Assoc 98:130-136

10. Winkley K, Stahl D, Chalder T, Edmonds ME, Ismall K (2007) Risk factors associated with adverse outcomes in a populationbased prospective cohort study of people with their first diabetic foot ulcer. J Diabetes its Complicat 21:341-349

11. Takahashi PY, Kiemele LJ, Chandra A, Cha SS, Targonski PV (2009) A retrospective cohort study of factors that affect healing in long-term care residents with chronic wounds. Ostomy Wound Manage 55:32-37

12. Walburn J, Vedhara K, Hankins M, Rixon L, Weinman J (2009) Psychological stress and wound healing in humans: a systematic review and meta-analysis. J Psychosom Res 67:253-271

13. Folkman S (1997) Positive psychological states and coping with severe stress. Soc Sci Med 45:1207-1221

14. Sultan S, Epel E, Sachon C, Vailant G, Hartemann-Heurtier A (2008) A longitudinal study of coping, anxiety and glycaemic control in adults with type 1 diabetes. Psychol Health 23:73-89 
15. Yi JP, Yi JC, Vitalioano PP, Weinger K (2008) How does anger control affect glycaemic control in diabetes patients? International J Behav Med 15:167-172

16. Kacerovsky-Bielesz G, Lienhardt S, Hagenhofer M et al (2009) Sex-related psychological effects on metabolic control in type 2 diabetes mellitus. Diabetologia 52:781-788

17. Blakytny R, Jude EB (2009) Altered molecular mechanisms of diabetic foot ulcers. Int J Low Extrem Wounds 8:95-104

18. Tarlton JF, Bailey AJ, Crawford E, Jones D, Moore K, Harding KD (1999) Prognostic value of markers of collagen remodeling in venous ulcers. Wound Repair Regen 7:347-355

19. Vedhara K, Miles J, Crown A et al (2007) Relationship of early childhood infections to adult cortisol in the Barry Caerphilly Growth (BCG) study. Psychoneuroendocrinology 32:865-873

20. Pruessner M, Hellhammer DH, Pruessner JC, Lupien SJ (2003) Self-reported depressive symptoms and stress levels in healthy young men: associations with the cortisol response to awakening. Psychosom Med 65:197-204

21. The Diabetes Control and Complications Trial Research Group (1993) The effect of intensive treatment of diabetes on the development and progression of long-term complications in insulin-dependent diabetes mellitus. N Engl J Med 329:977-986

22. Young MJ, Boulton AJM, Mcleod AF, Williams DRR, Sonksen PH (1993) A multicentre study of the prevalence of diabetic peripheral neuropathy in the UK hospital clinic population. Diabetologia 36:150-154

23. Zigmond AS, Snaith RP (1983) The hospital anxiety and depression scale. Acta Psychiatr Scand 67:361-370

24. Kendrick T, Dowrick C, McBride A et al (2009) Management of depression in UK general practice in relation to scores on depression severity questionnaires: analysis of medical record data. BMJ 338:b750

25. Feifel H, Strack S, Nagy VT (1987) Coping strategies and associated features of medically ill patients. Psychosom Med 49:616-625

26. Tarlton JF, Vickery CJ, Leaper DJ, Bailey AJ (1997) Postsurgical wound progression monitored by temporal changes in the expression of matrix metalloproteinase-9. Br J Dermatol 137:506-516

27. Tarlton JF, Knight PJ (1996) Comparison of reflectance and transmission densitometry, using document and laser scanners, for quantitation of stained Western blots. Anal Biochem 237:123-128
28. Tarlton JF, Knight PJ (1996) Clarification of immunoblots on polyvinylidene difluoride (PVDF) membranes for transmission densitometry. J Immunol Methods 191:65-69

29. Ince P, Kendrick D, Game F, Jeffcoate W (2007) The association between baseline characteristics and the outcome of foot lesions in a UK population with diabetes. Diabet Med 24:977-981

30. Pruessner JC, Wolf OT, Hellhammer DH et al (1997) Free cortisol levels after awakening: a reliable biological marker for the assessment of adrenocortical activity. Life Sci 61:2539-2549

31. Vedhara K, Tuinistra J, Miles JNV, Sanderman R, Ranchor AV (2006) Psychosocial factors associated with indices of cortisol production in women with breast cancer and controls. Psychoneuroendocrinology 31:299-311

32. Rice B, Kalker AJ, Schindler JV, Dixon RM (2001) Effect of biofeedback-assisted relaxation training on foot ulcer healing. J Am Podiatr Med Assoc 91:132-141

33. Miller GE, Cohen S, Rabin BS, Skoner DP, Doyle WJ (1999) Personality and tonic cardiovascular, neuroendocrine and immune parameters. Brain Behav Immun 13:109-123

34. de Groot J, Boersma WJA, Scholten JW, Koolhas JM (2002) Social stress in male mice impairs long-term antiviral immunity selectively in wounded subjects. Physiol Behav 75:277-285

35. Vreeburg SA, Hoogendijk WJG, van Pelt J et al (2009) Major depressive disorder and hypothalamic pituitary adrenal axis activity results from a large cohort study. Arch Gen Psychiatry 66:617-626

36. Osterberg K, Karslon B, Hansen AM (2009) Cognitive performance in patients with burnout, in relation to diurnal salivary cortisol. Stress 12:70-81

37. Yang EV, Bane CM, MacCallum RC, Kiecolt-Glaser JK, Malarkey WB, Glaser R (2002) Stress-related modulation of matrix metalloproteinase expression. J Neuroimmunol 133:144150

38. Prompers L, Schaper N, Apelqvist J et al (2008) Prediction of outcome in individuals with diabetic foot ulcers: focus on the differences between individuals with and without peripheral arterial disease. The Eurodiale Study. Diabetologia 51:747-755

39. Chesney MA, Chambers DB, Taylor JM, Johnson LM, Folkman S (2003) Coping effectiveness training for men living with HIV: results from a randomised clinical trial testing a group-based intervention. Psychosom Med 65:1038-1046 